\title{
Web OPEN-Integrated: Proposed Framework for Web Development*
}

\author{
Rashid Ahmad**, Zhang Li, and Farooque Azam \\ Software Engineering Institute, Beijing University of Aeronautics and Astronautics \\ No.37, XueYuan Road, HaiDian District, Beijing 100083, P.R. China \\ $\{r . a h m a d, 1 i l y$, farooque\}@buaa.edu.cn
}

\begin{abstract}
Most Web applications are designed in an ad-hoc manner. Web applications, due to their peculiar nature, cannot be just developed as conventional software projects. Haire et al advocate Web OPEN [1] [2], extension to OPEN (Object-oriented Process, Environment, and Notation), as suitable model and provide a good study on it. We however, argue Web OPEN does address some of the peculiar issues of Web applications but not all, which we identify in this paper. We then propose a hypothesis that Web OPEN can be augmented in its efficacy with integration of components from other methodologies. In this paper we have investigated such components that could serve our hypothesis. We name the new model as Web OPEN Integrated.
\end{abstract}

\section{Introduction}

OPEN (Object-oriented Process, Environment, and Notation) is a process-focused methodological approach to software-intensive systems development useful for both object-oriented and Component-Based Development (CBD). OPEN was developed and is maintained by the not-for-profit OPEN Consortium, an international group of methodologists, CASE tool vendors and developers. It is documented in a series of books, papers (e.g. [1], [2], [3], [4], [5], [6], [7]) and in many journal articles, particularly in the journal JOOP. A unique aspect of OPEN is that it is not merely a process but a configurable family of processes, defined in terms of a metamodel (Fig. 1), known as the OPEN Process Framework (OPF). From these instances of the process fragments, organizationally-specific processes can be readily constructed. The component-based nature of OPEN permits appropriate extensions to support development in new domains. One such set of extensions is those for Web development, called Web OPEN (see Haire et al. [1], [2] for a detailed discussion on Web OPEN).

\section{Some Important Issues Not Addressed by Web OPEN}

In this section we will briefly discuss few important issues specific to WBA (web based applications) that have not been addressed in the Web OPEN.

- Quality Assurance for Documentation: When an engineering manual or the content of the website has an error or foggy expression, the quality is lowered because that manual/content is ultimately read by millions of readers. Good techni-

\footnotetext{
Supported by Beijing Municipal Science \& Technology New Star Plan (H013610270112)

** Corresponding author: Tel: +86-13691144459
} 
cal documentation can help people avoid common mistakes and errors. Web OPEN does emphasize on documentation but does not say how.

- Process Variation: Web applications are software intensive, and for software, process variation can never be eliminated or even reduced below a moderate level. No two modules are the same, so process performance always includes an intrinsic degree of variability. There are large differences in skills and experience from one developer to another. Web OPEN does not address this issue.

- Increased Importance of "Quality" Attributes and "Security": We discuss security and quality together because we believe both are interrelated. They address this issue with task define website testing strategy which emphasizes on whether the users can see the website concurrently or not. Another task they have "define website standards" which emphasize on consistency. We argue that quality and security being the most important issues have not been dealt with effectively.

- Navigation Design: Once the WBA architecture has been established and the components of the architecture have been identified, the designer must define Navigation Pathways that enable a user to access WBA contents and services. Web OPEN addresses this with task "create navigation map for website" but we argue that creating this map is broad term and we have to perform many subtasks before we are able to create navigation map for the website. This issue has not been addressed in Web OPEN.

- Short Time Frames for Initial Delivery: We believe this is the major driving force behind the ad-hoc ism in website development. This is creating a big gap between the commercial practice in this area and the researcher's direction. Although they say this issue has been indirectly addressed in activity "Website management" but we believe this needs to be addressed explicitly so that the clients are convinced they would reach to the market in time.

- Process Improvement Component: Room for improvement always exists in software processes. Despite this fact, OPF and Web OPEN do not have any component for improvement of processes.

\section{Proposed Hypothesis: Web OPEN-I (Web OPEN Integrated)}

Web OPEN which specifically focuses on Web development is handicapped with some limitations and is unable to address some of the peculiar issues associated with Website development. Our hypothesis focuses to cure it by integrating it with another well established methodology. We have selected Six Sigma and we contend that we can augment the efficacy of Web OPEN by integrating it with some components/tools from "six sigma for software".

\subsection{Overview of Six Sigma for Software}

Originated at Motorola in mid 80's, Six Sigma is a business-driven, multi-faceted approach to process improvement, reduced costs, and increased profits. With a fundamental principle to improve customer satisfaction by reducing defects, its ultimate performance target is virtually defect-free processes and products (3.4 or fewer defective parts per million (ppm) or 99.9997 percent defect free). The Six Sigma methodology, consisting of the steps "Define, Measure, Analyze, Improve, Control 
(DMIAC)" is the roadmap to achieving this goal. Within this improvement framework, it is the responsibility of the improvement team to identify the process, the definition of defect, and the corresponding measurements. This degree of flexibility enables the Six Sigma method, along with its toolkit, to easily integrate with existing models of software process implementation.

When and how does a DMAIC Project Start? A DMAIC project is an opportunity to improve a process. So it starts when we identify that a business process needs improvement. How do we know when a business process needs improvement? There can be various sources, for example,

- We have a process that the customer is complaining about very often then resolving the problem, because of which customer is complaining, is a basis to improve our process.

- Our process is not meeting its service level.

\subsection{Connecting Web OPEN and Six Sigma}

Due to the existing incredible synergy between Web OPEN and Six Sigma, DMIAC can be very well integrated to the metamodel of OPF. Fig. 2 (for clear readability of the figures, please zoom to $\mathbf{2 0 0 \%}$ ) shows our proposed integrated metamodel of OPF in UML. The producer can invoke DMIAC project anytime when needed. Circumstances when we invoke DMIAC project, has been explained in section 3.1 above. The out put of this process is the product that has to be delivered.

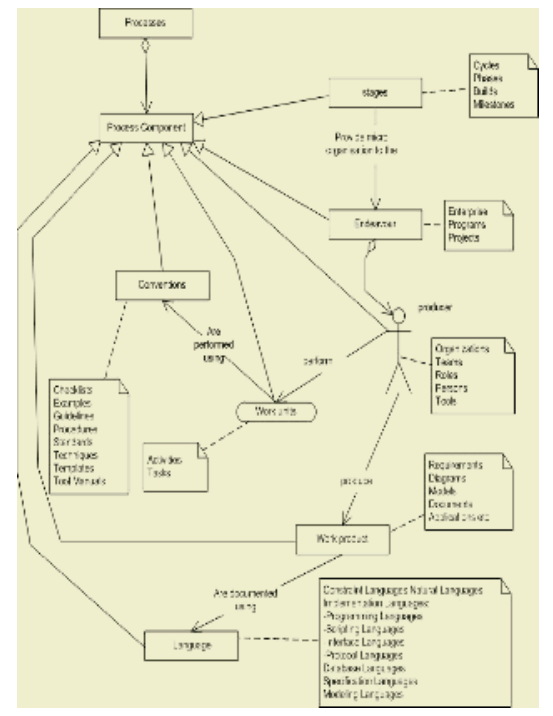

Fig. 1. Original OPF metamodel

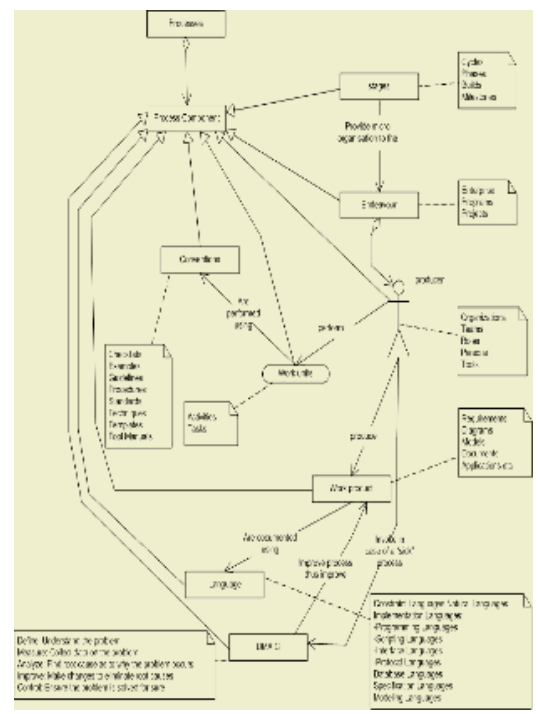

Fig. 2. Proposed OPF metamodel

\section{How Web OPEN-I Addresses the Issues Discussed in Section 2}

The integration of components from "six sigma for software" enables us to propose new Tasks and Techniques for Web OPEN. In total we have proposed following 3 
new tasks, 7 new sub tasks and 1 new technique. Below (in Table 1) we have listed the issues and the proposed tasks, subtasks and techniques that will address them.

Table 1. New Tasks, Subtasks and Techniques

\begin{tabular}{|c|c|}
\hline Issues & Addressed by \\
\hline $\begin{array}{l}\text { - Quality assurance for } \\
\text { documentation }\end{array}$ & $\begin{array}{l}\text { - New task "Do Document" (fig.5) } \\
\text { - New technique "Controlled Natural Languages" (fig. 6) }\end{array}$ \\
\hline - Process Variation & - New task "Measure Process Variation" \\
\hline $\begin{array}{l}\text { - Increased importance of } \\
\text { "Quality" attributes and } \\
\text { "Security" }\end{array}$ & $\begin{array}{l}\text { - New subtask "Create CSS standards" } \\
\text { - New subtask "Validate the web" } \\
\text { - New subtask "Comply with WAI - The Web Accessibility Initiative" } \\
\text { - New process component DMIAC concentrates on "Reducing De- } \\
\text { fects" with a goal of } 99.9997 \text { percent defect free system (not only } \\
\text { defects from code but also other defects like deign defects etc) }\end{array}$ \\
\hline - Navigation Design & $\begin{array}{l}\text { - New subtask "Identify semantics of navigation" } \\
\text { - New subtask "Define mechanics (syntax) for navigation" } \\
\text { - New subtask "Create navigation conventions" } \\
\text { - New subtask "Deign navigation aids" }\end{array}$ \\
\hline $\begin{array}{l}\text { - Short time frames for } \\
\text { initial delivery }\end{array}$ & - New task "Create "time to market' plan" \\
\hline $\begin{array}{l}\text { - Process improvement } \\
\text { component }\end{array}$ & $\begin{array}{l}\text { - New process component (DMAIC) added to the OPF metamodel } \\
\text { (see fig. } 2 \text { ) }\end{array}$ \\
\hline
\end{tabular}

Due to the limitation of the space we are unable to explain all the new tasks, sub tasks and techniques. We will however (as sample) explain task "Do Document" and technique "Control Natural Language" to show how it will address the issue of "Quality assurance for documentation" in WBA. Explanation of other tasks and subtasks will be published elsewhere.

\subsection{Task "Do Document" and Technique "Controlled Natural Languages" Explained}

For quality assurance in technical documentation we have proposed one new task "Do Document" (Fig. 3) and one new technique "Controlled Natural Languages" (Fig. 4). Let's have a look at it.

\section{Conclusion and Future Work}

The Software Process Engineering Metamodel (SPEM) recently adopted technology by OMG's, is a metamodel for defining processes and their components. This metamodel is used to describe a concrete software development process or a family of related software development processes. The SPEM specification is structured as a UML profile, and provides a complete MOF-based metamodel. This approach facilitates exchange with both UML tools and MOF-based tools/repositories. The MetaObject Facility (MOF) is the OMG's adopted technology for defining metadata and representing it as CORBA objects. The deliverables of OPEN can be represented in any notation like OML, UML etc. However there are some aspects of OPEN that can be expressed readily in OML but cannot be expressed or will not be properly expressible within the semantics of UML (e.g. responsibilities, rule sets, exceptions etc) [4]. 


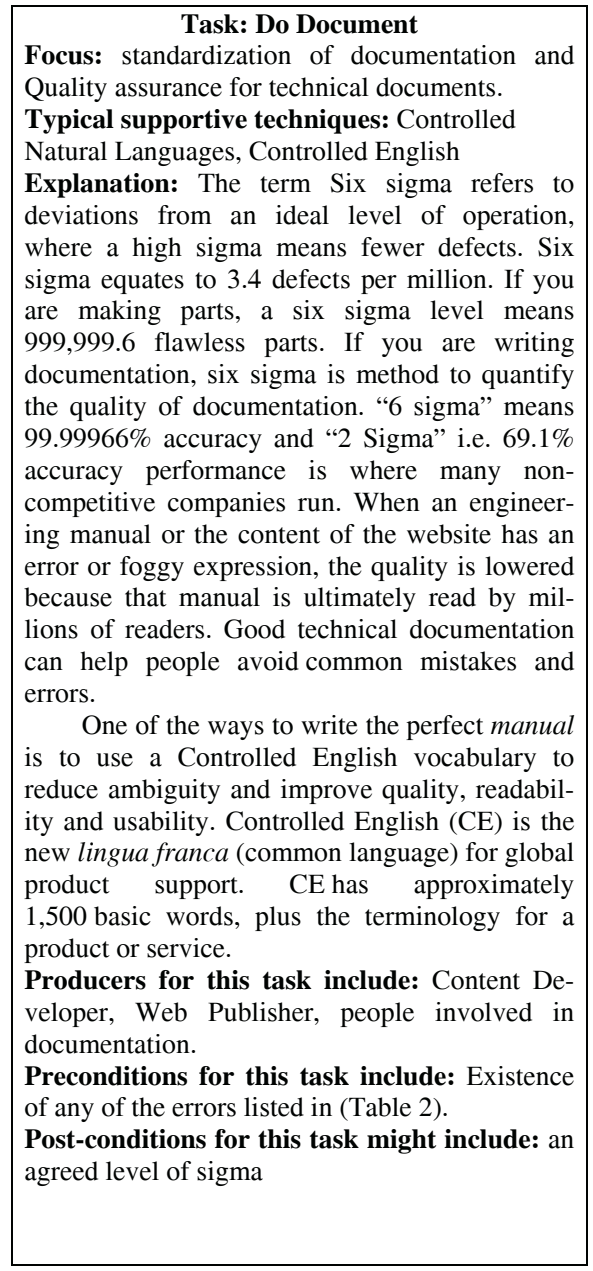

Fig. 3. Task "Do Document"

\begin{tabular}{l} 
Technique: Controlled Natural Languages \\
Focus: Minimizing errors in documentation, \\
standardization of documentation. \\
Typical tasks for which this is needed: Do \\
Document \\
Technique description: A controlled natural \\
languages technique can be thought of learning a \\
different language. The aim of the technique is to \\
minimize the errors in written documents and \\
contents. Using this technique is particularly \\
useful in web development as by nature they are \\
content driven. \\
Typical supportive tools: Acrocheck \\
(http://www.acrolinx.de ), CLAT (Controlled \\
Language Authoring Technology) \\
(http://www.iai.uni-sb.de/iaide/de/clat.htm), \\
MAXit - Controlled English Checker \\
http://www.smartny.com \\
Technique usage: This technique can be used by \\
following simple steps for six sigma standard. \\
- Create a CE vocabulary for your company \\
(tools such as SMART Text Miner can be used \\
for the rapid development of a CE vocabulary) \\
- Teach the writers how to use Controlled Eng- \\
lish. \\
- Use English-to-Metric converter to avoid \\
conversion errors. Use available tools to find \\
the errors in the text \\
- Check currencies, quantities, qualities, sizes, \\
URLs \\
- Use tool metrics to calculate the percentage of \\
error. \\
- Validate signal names, instrument labels and \\
Deliverables and outputs (post-condition): A \\
document that demonstrates a high standard of \\
error free and ambiguity free documentation. \\
\hline
\end{tabular}

Fig. 4. Technique "Controlled Natural Languages"

Table 2. Common errors in document

- Spelling errors
- Foggy writing with confused gobbledygook/
jargon
- Long sentences that are more than 21 words
- Key information is missed, confused or omitted
- "Failure to Warn" missing or wrong warnings
- Wrong or non-existent page number references
- Incorrect labels/references for instrumentation
- Content is nice to know, not need to know
- Wrong/multiple names for parts or nomenclature
- Texts written by engineers in "engineering-ese"

- Measurements are vague, missed or wrong

- Use of -ing words that cause English ambiguity

-Wrong metric conversions/omitted dual dimensions

- Obsolete telephone numbers, URLs, product names

-Wrong or missing illustrations, graphics, callouts - Invention of terminology for writer convenience

- Multiple meanings for the same abbreviation

- Acronyms that do not follow global standards

- Tools/materials listed are not available worldwide

- Failure to edit/review texts for technical accuracy 
Therefore to comply with the widely accepted OMG's standards, the future work includes: making OPF as MOF compliant, and Mapping between SPEM and OPF/Web OPEN-I.

\section{References}

1. B. Haire, B. Henderson-Sellers, and D. Lowe: "Supporting web development in the open process: additional tasks," in COMPSAC'2001: International Computer Software and Applications Conference, Chicago, Illinois, USA, Submitted, IEEE Computer Society

2. Henderson-Sellers, B., Lowe, D., \& Haire, B.: OPEN Process Support for Web Development. Annals of Software Engineering, 13, 163-201,2002

3. I. Graham, B. Henderson-Sellers, and H. Younessi: The OPEN Process Specification, Addison-Wesley, 1997

4. B. Henderson-Sellers, A. Simons, and H. Younessi: The OPEN Toolbox of Techniques, Addison-Wesley, UK, 1998

5. D. Firesmith, G. Hendley, S. Krutsch, and M. Stowe: Object-Oriented Development Using OPEN: A Complete Java Application, Addison-Wesley, Harlow, UK, 1998

6. B. Henderson-Sellers and B. Unhelkar: OPEN Modeling with UML, Addison-Wesley, Harlow, UK, 2000

7. D. Firesmith and B. Henderson-Sellers: The OPEN Process Framework. An Introduction, Addison-Wesley, Harlow, UK, 2001 